\title{
Polymer Scaffolds with Preferential Parallel Grooves Enhance Nerve Regeneration
}

\author{
Atefeh Mobasseri, PhD, ${ }^{1,2}$ Alessandro Faroni, PhD, ${ }^{1}$ Ben M. Minogue, PhD, \\ Sandra Downes, $\mathrm{PhD},{ }^{2}$ Giorgio Terenghi, $\mathrm{PhD},{ }^{1}$ and Adam J. Reid, $\mathrm{PhD}^{1}$
}

We have modified the surface topography of poly $\varepsilon$-caprolactone (PCL) and polylactic acid (PLA) blended films to improve cell proliferation and to guide the regeneration of peripheral nerves. Films with differing shaped grooves were made using patterned silicon templates, sloped walls (SL), V-shaped (V), and square-shaped (SQ), and compared with nongrooved surfaces with micropits. The solvent cast films were tested in vitro using adult adiposederived stem cells differentiated to Schwann cell-like cells. Cell attachment, proliferation, and cell orientation were all improved on the grooved surfaces, with SL grooves giving the best results. We present in vivo data on SpragueDawley rat sciatic nerve injury with a 10-mm gap, evaluating nerve regeneration at 3 weeks across a polymer nerve conduit modified with intraluminal grooves (SL, V, and SQ) and differing wall thicknesses (70, 100, 120, and $210 \mu \mathrm{m})$. The SL-grooved nerve conduit showed a significant improvement over the other topographical-shaped grooves, while increasing the conduit wall thickness saw no positive effect on the biological response of the regenerating nerve. Furthermore, the preferred SL-grooved conduit (C) with $70 \mu \mathrm{m}$ wall thickness was compared with the current clinical gold standard of autologous nerve graft $(\mathrm{Ag})$ in the rat 10-mm sciatic nerve gap model. At 3 weeks postsurgery, all nerve gaps across both groups were bridged with regenerated nerve fibers. At 16 weeks, features of regenerated axons were comparable between the autograft (Ag) and conduit (C) groups. End organ assessments of muscle weight, electromyography, and skin reinnervation were also similar between the groups. The comparable experimental outcome between conduit and autograft, suggests that the PCL/PLA conduit with inner lumen microstructured grooves could be used as a potential alternative treatment for peripheral nerve repair.

\section{Introduction}

$\mathbf{R}$ EPAIR OF A PERIPHERAL nerve gap is a clinical problem for which a bioengineered solution is being sought. An effective nerve conduit needs to provide a supportive environment for cellular interactions promoting axonal regeneration across a nerve gap.

Biodegradable polymers such as poly $\varepsilon$-caprolactone (PCL) and polylactic acid (PLA) are promising materials toward development of innovative nerve conduits. ${ }^{1-3}$ The blend of these polymers has been studied to tailor crucial properties, including mechanical strength and hydrophobicity/hydrophilicity. ${ }^{4}$ We have previously reported that ultrathin PCL films and PCL/PLA blended films with a micropitted surface are able to support the attachment and growth of the NG108-15 neuronal cell line and Schwann cells. ${ }^{5}$ Schwann cells are key players in nerve regeneration through delivery of neurotrophic factors and extracellular matrix proteins; however, the absence of Schwann cells in a nerve gap has led to much current work being focused on the use of stem cells to deliver this. We have previously demonstrated that PCL films can support adipose-derived stem cells (ASCs), differentiated to a Schwann cell-like phenotype (dASC), as a potential tissue-engineered route to nerve repair. ${ }^{6}$ Furthermore, we have established that the PCL/PLA micropitted films can be used as bioresorbable nerve guidance conduits for bridging peripheral nerve gaps in an in vivo model. ${ }^{2}$ Hence, PCL/PLA polymer films offer a viable alternative to the surgical approach of using the nerve autograft, which at present is the gold standard for peripheral nerve reconstruction. The use of synthetic, off-the-shelf polymer conduits would avoid the problems associated with nerve grafting, such as lack of autograft tissue, donor site morbidity, and increased surgical time. However, the surface and the material properties of the biodegradable polymers could be designed and engineered with specific topographical cues to improve the cellular interaction with the biomaterial and to provide the optimal environment for peripheral nerve regeneration.

\footnotetext{
${ }^{1}$ Blond McIndoe Laboratories, Centre for Tissue Injury and Repair, Institute of Inflammation \& Repair, University of Manchester, Manchester, United Kingdom.

${ }^{2}$ Material Sciences Centre, School of Materials, University of Manchester, Manchester, United Kingdom.

(C) Atefeh Mobasseri et al. 2015; Published by Mary Ann Liebert, Inc. This Open Access article is distributed under the terms of the Creative Commons License (http://creativecommons.org/licenses/by/4.0), which permits unrestricted use, distribution, and reproduction in any medium, provided the original work is properly credited.
} 
Surface topography of biomaterials is known to have a significant impact on the morphology and cytoskeletal organization. ${ }^{7}$ Several studies have shown that the topographic and dimensional properties of scaffolds exert a range of effects, including the attachment, spreading, growth, differentiation, and transcription profile of the cells. ${ }^{7-10}$ Surface modifications have a similar effect on cells of the peripheral nerve, where the alignment of Schwann cells plays an important role in the regeneration of damaged nerves. It has been shown that Schwann cells align successfully along biodegradable polyDL-lactic acid microgrooves coated with laminin. ${ }^{11}$ Using Schwann cells and dorsal root ganglion neuronal cocultures, the same authors also demonstrated that these substrates were able to direct the growth and alignment of the neurites sprouting from the neuronal cells. ${ }^{12}$ Schwann cells were shown to align on microgroove PLA substrates, and these polymer microgroove conduits were suggested to enhance peripheral nerve regeneration when compared with smooth conduit controls. ${ }^{13}$

The pivotal function of Schwann cells in nerve growth and regeneration is well established, ${ }^{14,15}$ and it has been illustrated that Schwann cell transplantation into a nerve gap may enhance axonal regeneration. ${ }^{16,17}$ However, due to the lengthy procedure of isolating and culturing Schwann cells, an alternative treatment has been sought in the form of stem cells differentiated toward the Schwann cell phenotype. ${ }^{18,19}$ The phenotype of these transdifferentiated cells has been confirmed alongside improvement in outgrowth and myelination of neurites in vitro in the presence of dASCs. ${ }^{19}$ In vivo studies have also indicated improved axonal regeneration when fibrin conduits were treated with Schwann cells or dASCs. ${ }^{20}$

We have recently developed and optimized the PCL/PLA biodegradable polymer conduit with a unique microgrooved design, which can support the attachment and proliferation of neural cells. ${ }^{21}$ In the present study, we assess the compatibility of dASCs on the microgrooved films in vitro, and evaluate the in vivo performance of the patterned biomaterial as a nerve conduit in a rat sciatic nerve gap model.

\section{Materials and Methods}

\section{Film preparation}

Films were produced by casting a $3 \% \mathrm{wt} / \mathrm{v}$ blend of PCL (Mn 70,000-90,000 g/mol) and PLA (Mn 30,000-60,000 g/ mol) (4:1 PCL/PLA) (Sigma-Aldrich) in dichloromethane
(99\% purity) (Fisher Scientific) on silicon substrates. Grooved films were prepared on patterned silicon substrates created by photolithography, wet, and dry etching (substrates obtained as described in a previous study ${ }^{21}$ ). Three patterned variations of silicon substrates were used to prepare PCL/ PLA films with different microgroove conformation, sloped walls (SL), V-shaped (V), and square-shaped (SQ), with a depth of the grooves in all three shapes of $5 \mu \mathrm{m}$ (Fig. 1). The SL-shaped grooves had a width in a range of $15-20 \mu \mathrm{m}$ and ridges in the range of $3-6 \mu \mathrm{m}$, while the $\mathrm{V}$ grooves had a width in a same range $(15-20 \mu \mathrm{m})$, but no ridges between adjacent grooves. The angle of the wall was $40^{\circ}-50^{\circ}$ in both SL-shaped and V grooves. The SQ grooves had upright walls $\left(90^{\circ}\right)$ with a $10 \mu \mathrm{m}$ width and ridges of $10 \mu \mathrm{m}$ (Fig. 1). Control films were also prepared on a smooth silicone substrate, whereby the flat uppermost surface of the film resulted in a micropitted conformation. ${ }^{5}$

\section{Conduit preparation}

Film was rolled around a cannula (16-gauge size) and sealed using a $40^{\circ} \mathrm{C}$ hot plate. Conduits had an inner diameter of $1.6 \mathrm{~mm}$ and a length of $14 \mathrm{~mm}$. The hot sealing temperature was selected to seal the conduit with minimum effect on the intraluminal structure. Conduits were sterilized for in vivo experiments with UV radiation for $60 \mathrm{~min}$ in a microbiological safety cabinet (Envair). Three sets of conduits were prepared for three separate in vivo experiments as follows:

(1) Comparing the grooved conduits with different groove shapes;

Conduits were made of thin PCL/PLA films (wall thickness of $70 \mu \mathrm{m}$ ) with grooved (SL, V) and nongrooved (pitted) intraluminal structure.

(2) Comparing the SL-grooved conduits with different wall thickness;

SL-grooved conduits with different wall thickness and improved mechanical strength were prepared. SL-shaped grooved films were chosen because of the promising results obtained with the initial in vivo experiments (cf. In Vivo Experiments section). Four groups of samples were prepared as follows (Fig. 2A):

- Group 1: Thin one layer conduit (with an average wall thickness of $70 \mu \mathrm{m})$.

- Group 2: Thick one layer conduit (with an average wall thickness of $100 \mu \mathrm{m})$.

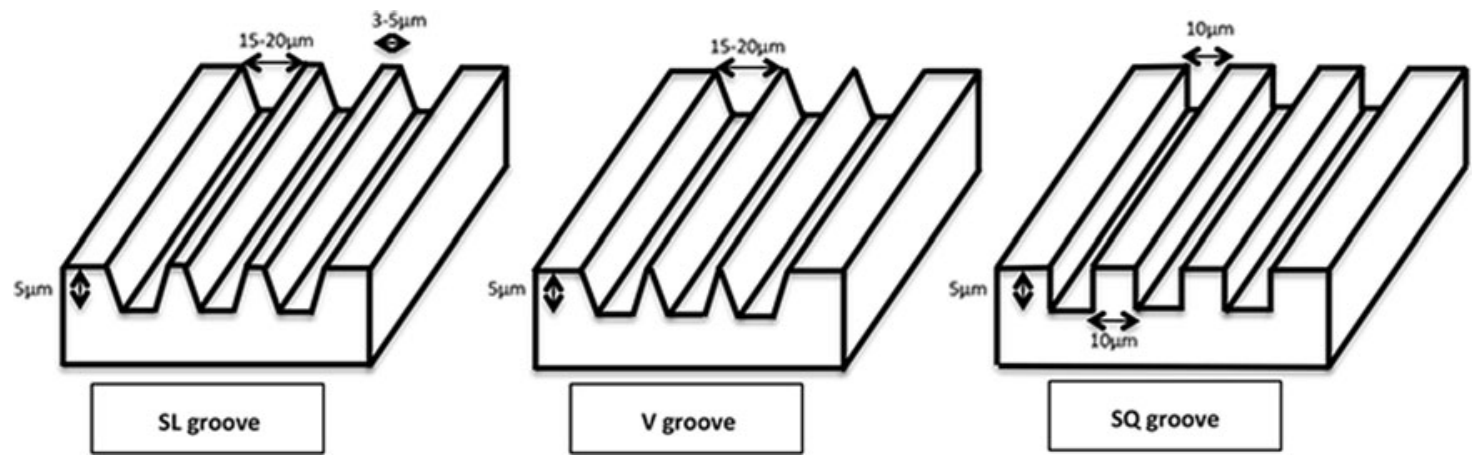

FIG. 1. The schematic figures of the SL, V, and SQ-grooved films show the grooved structure specifications. SL, sloped walls; V, V-shaped; SQ, square-shaped. 
A

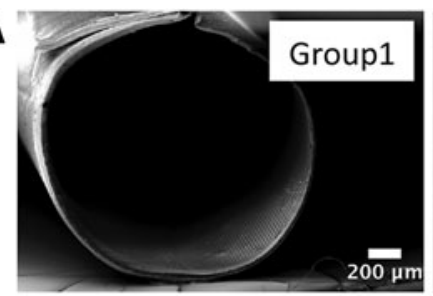

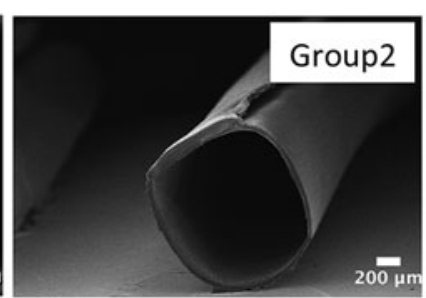
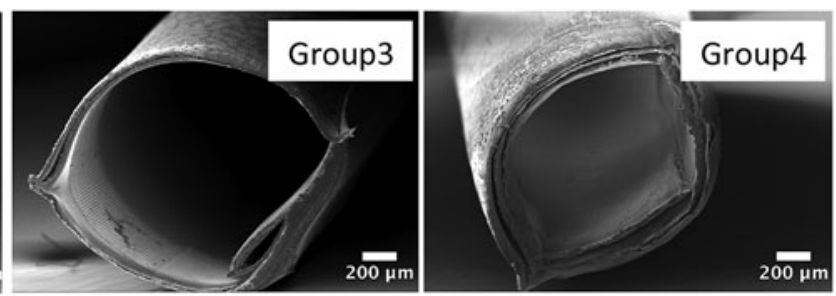

B

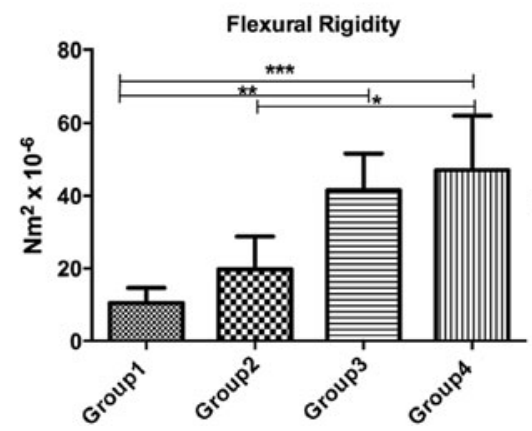

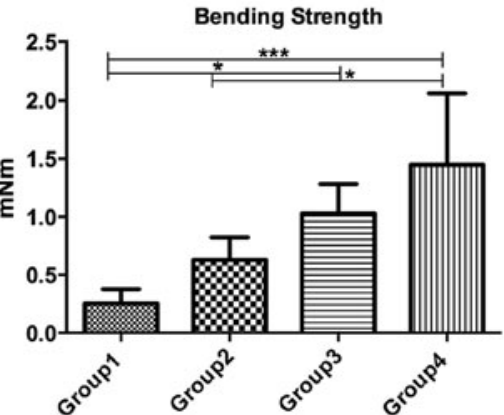

Group1: Thin one layer conduit (Wall thickness $70 \mu \mathrm{m}$ )

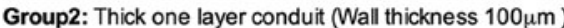

Group3: Spiral layer conduit (Wall thickness $210 \mu \mathrm{m}$ )

Group4: Multilayers conduit (wall thickness $130 \mu \mathrm{m}$ )

FIG. 2. (A) SEM images of the conduits; group 1: thin one layer conduit, with average wall thickness of $70 \mu \mathrm{m}$; group 2 : thick one layer conduit, with average wall thickness of $100 \mu \mathrm{m}$; group 3: spiral layered conduit, with average wall thickness of $210 \mu \mathrm{m}$; group 4: multilayered conduit, with average wall thickness of $130 \mu \mathrm{m}$. (B) Flexural rigidity and bending strength of conduits with differing wall thickness. $n=3(* p<0.05, * * p<0.01 * * * p<0.001)$. SEM, scanning electron microcopy.

- Group 3: Spiral layered conduit (with an average wall thickness of $210 \mu \mathrm{m}$ );

One thin film with $70 \mu \mathrm{m}$ thickness spirally rolled in three layers. To reduce the space between layers, a hot needle (diameter: $0.6 \mathrm{~mm}$ ) was used to press the layer from the inside of the conduit in three areas, but maintaining the groove topology of the inner surface.

- Group 4: Multilayered conduit (with an average wall thickness of $130 \mu \mathrm{m}$ ); Initially two smooth films and one grooved film were bound together using a $40^{\circ} \mathrm{C}$ hot plate, with the thickness of the three-layered film of $130 \mu \mathrm{m}$. The three-layered film was used to prepare the conduit with a grooved topology of the inner surface and a smooth outer surface.

(3) Comparing the SL-grooved conduit with autograft;

The SL-grooved conduits with $70 \mu \mathrm{m}$ wall thickness were prepared for both the short-term and long-term in vivo experiment.

\section{Scanning electron microscopy imaging}

Before imaging with scanning electron microcopy (SEM) (VPSEM, Zeiss EVO60; Carl Zeiss Ltd., with 5KV accelerating voltage), samples were mounted on aluminum stubs and gold coated for 2 min by vacuum sputtering (Edward). Conduits were opened and mounted on the SEM stub and gold coated to examine the structure of grooves on the intraluminal surface post in vivo experiments. Desktop SEM (Phenom World G2) was used to image the inner surface of the conduit after 3 and 16 weeks of implantation.

\section{Three-point bending test}

The conduits with different wall thicknesses (group 1-4) (cf. Conduit Preparation section) were tested for a threepoint bending test by an Instron $1122(n=5)$ to analyze flexural rigidity and bending strength. The speed was set at $5 \mathrm{~mm} / \mathrm{min}$ with the force load of $10 \mathrm{~N}$ and the span of $20 \mathrm{~mm}$. The resistance to the bending and deformation (flexural rigidity) and the maximum strength the conduit could endure before buckling (bending strength) were measured with Instron Series IX (version 8.12.00).

\section{ASC preparation}

ASCs were isolated and cultured according to the procedure explained by Kingham et al. ${ }^{19}$ Briefly, fat pads were harvested from rats (according to the UK Animal Scientific Procedure Act 1986) and were enzymatically digested in $0.15 \%(\mathrm{w} / \mathrm{v})$ collagenase type I (Invitrogen). The tissue was filtered $(70 \mu \mathrm{m}$ filter), centrifuged, and then cultured in a flask containing alpha minimum essential media (Invitrogen), $10 \%$ fetal bovine serum, and $1 \%$ penicillin-streptomycin (Invitrogen). Differentiation of stem cells to Schwann cell phenotype was carried out adapting the procedure used previously. ${ }^{19}$ The medium containing $1 \mathrm{mM} \beta$-mercaptoethanol (Sigma-Aldrich) was added to the ASC culture at passage 2 and incubated for $24 \mathrm{~h}$ at $37^{\circ} \mathrm{C}$ and $5 \% \mathrm{CO}_{2}$. Then, cells were incubated with fresh medium supplemented with $35 \mathrm{ng} / \mathrm{mL}$ all-trans-retinoic acid for $72 \mathrm{~h}$ at $37^{\circ} \mathrm{C}$ and $5 \%$ $\mathrm{CO}_{2}$. Following washes, cells were incubated in the differentiation medium containing $5 \mathrm{ng} / \mathrm{mL}$ platelet-derived growth factor (PeproTech Ltd.), $10 \mathrm{ng} / \mathrm{mL}$ basic fibroblast growth factor (PeproTech Ltd.), $14 \mu \mathrm{M}$ forskolin, and $252 \mathrm{ng} / \mathrm{mL}$ GGF-2 for further 2 weeks. Kingham et al. showed that the differentiated ASC prepared with this protocol expressed the Schwann cell markers, including S100, $\mathrm{p} 75$, and glial fibrillary acidic protein (GFAP). ${ }^{19}$

\section{In vitro testing}

Cell attachment and alignment were tested on pitted and grooved PCL/PLA films using dASCs ( $n=3$ each group). PCL/PLA films were immobilized using CellCrown ${ }^{\mathrm{TM}}$ 
(Scaffdex) culture inserts in 12-well plates. dASCs were plated on the films $(10,000$ cells $/ \mathrm{mL})$ and cultured for up to 8 days.

For cell attachment tests, at $24 \mathrm{~h}$ postseeding, the adherent cells were washed with PBS and fixed with $4 \%$ paraformaldehyde/phosphate-buffered saline (PFA/PBS). Following one wash with PBS, cells were stained with Alexa Fluor $488 \AA$ phalloidin (Invitrogen) according to the manufacturer's instructions. The films with adherent cells were finally mounted onto coverslips using Vectashield containing 4',6diamidino-2-phenylindole dihydrochloride (DAPI) (Vector Laboratories) to stain the cell nuclei. Images of the stained cells were taken using a fluorescence microscope (Olympus) and Image ProPlus software (v 6.0) (Media Cybernetics). Images of the cells in five separate fields of view were captured for each film sample, and cell numbers calculated using Image J (1.47f) software (National Health Institute).

Cell proliferation experiments were carried out using the AlamarBlue ${ }^{\circledR}$ assay (ABD Serotec) after culturing the cells on the different types of PCL/PLA-grooved films for up to 8 days. Absorbance readings, which give an indication of the number of cells, were taken at $570 \mathrm{~nm}$ and $600 \mathrm{~nm}$ using a spectrophotometer and calculated as percentage reduction of AlamarBlue as per the manufacturer's protocol. All experiments were performed in triplicate.

\section{In vivo testing}

Three sets of in vivo experiments were performed to evaluate the optimized structure for nerve guide conduits as follows:

(1) Short-term (3 weeks) study comparing SL, V, and SQ-grooved conduits with nongrooved conduits

(2) Short-term (3 weeks) study comparing the SLgrooved conduit with wall thicknesses of 70,100 , 120 , and $210 \mu \mathrm{m}$

(3) Short-term (3 weeks) and long-term (16 weeks) studies comparing the SL-grooved conduit with autograft

All the work was conducted in keeping with the terms of the Animals Scientific Procedures Act 1986, United Kingdom. Young, adult male Sprague-Dawley rats (weighing 180-220 g; Harlan) were anesthetized by inhalational of isofluorane (Abbott Laboratories). The left sciatic nerve of the rat was exposed and a section was removed to leave a 10-mm gap. Under an operating microscope (Zeiss), the proximal and distal nerve stumps of the transected nerve were secured within the $14 \mathrm{~mm}$ long, single-layered PCL/ PLA conduits with and without grooves $(n=6$ rats each conduit type) using two epineurial sutures (9.0 Ethilon) at either ends. Both nerve stumps were positioned $2 \mathrm{~mm}$ from the conduit ends to ensure that the proximal and distal nerve ends were separated by a 10-mm gap. The muscle and skin were closed and sutured in layers, and nerve regeneration was allowed to proceed for the periods defined above. A similar surgical approach was used for the nerve repair with conduits with different wall thicknesses (group 1-4, $n=6$ each group) (cf. Conduit Preparation section), and the rats were kept for 3 weeks postoperatively. For the autologous nerve graft group, $10 \mathrm{~mm}$ of sciatic nerve was excised, reversed, and sutured with 9/0 Ethilon epineurial sutures. Each animal was housed in cages of four after surgery and exposed to 12-h light/ 12-h dark cycles, with liberal access to food and water.

\section{Tissue harvesting}

Short-term study. After 3 weeks of implantation, the animals were euthanized and autograft and conduits (including the regenerated nerves) were harvested together with a $2 \mathrm{~mm}$ length of proximal and distal nerve. PFA (4\%) was used to fix the tissue for $24 \mathrm{~h}$, followed by washes in PBS containing $15 \%$ sucrose and $0.1 \%$ sodium azide for 3 consecutive days.

Long-term study. After 16 weeks of implantation, the animals were anesthetized deeply with isofluorane and perfusion fixation was performed. First, $0.9 \%$ heparinized saline $\left(0.9 \% \mathrm{NaCl}, 50 \mathrm{U} / \mathrm{mL}\right.$ heparin) at $4^{\circ} \mathrm{C}$ and then $4 \%$ paraformaldehyde in $0.1 \mathrm{M}$ PBS were perfused transcardially. Then, the regenerated nerves and conduits were collected. The gastrocnemius muscles and skins of the footpads of both contralateral and operated sides were also harvested. All tissues were postfixed in PFA (4\%) for $24 \mathrm{~h}$, followed by three washes in PBS containing $15 \%$ sucrose and $0.1 \%$ sodium azide.

Following tissue harvesting and fixation, the conduits were opened and the regenerated nerves were removed under a dissecting microscope. The tissue was embedded in an

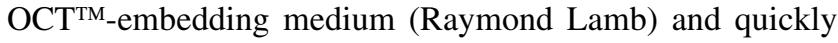
frozen in liquid nitrogen and stored at $-40^{\circ} \mathrm{C}$ freezer.

\section{Immunohistochemistry}

Tissue sections were cut as described (cf. Quantification of Nerve Regeneration for Short-Term Experiments section) before being permeabilized in a $0.2 \%$ Triton X-100/PBS solution for $1 \mathrm{~h}$ at room temperature. After further washes in PBS $(2 \times 5 \mathrm{~min})$, blocking serum was added for $1 \mathrm{~h}$ at room temperature. Sections were incubated in primary antibody at $4^{\circ} \mathrm{C}$ overnight, washed twice in PBS for $5 \mathrm{~min}$, incubated with secondary antibody for $2 \mathrm{~h}$ at room temperature in the dark, with two further washes with PBS for $5 \mathrm{~min}$, and finally mounted under coverslips using the mounting media fluorescence with DAPI (Vector Laboratories).

\section{Quantification of nerve regeneration for short-term experiments}

The conduits were carefully removed from the regenerated nerves, which were then prepared for cryostat sectioning by freezing the samples in liquid nitrogen. Longitudinal $15-\mu \mathrm{m}$-thick sections were collected onto glass slides coated with Vectabond (Vector Laboratories) and dried overnight at $37^{\circ} \mathrm{C}$ to improve adherence. Immunostaining was carried out using antibodies to the axonal marker PGP9.5 (rabbit polyclonal 1:200 dil; Dako) incubated overnight at $4^{\circ} \mathrm{C}$. Following washes in PBS $(3 \times 10 \mathrm{~min})$, the sections were incubated with FITC-conjugated secondary antibodies (anti-rabbit IgG 1:100 dil; Vector Laboratories) for $1 \mathrm{~h}$ in the dark at room temperature. After further washes in PBS, the sections were mounted with Vectashield containing DAPI (Vector Laboratories). 
The amount of regenerating axons was assessed on immunostained nerve sections using an Olympus fluorescence microscope and Image ProPlus software (Media Cybernetics). Overlapping images were taken across the width of the immunostained nerve sections using a $4 \times$ objective. For each nerve sample, images were taken on three nonconsecutive longitudinal sections cut at the midpoint of the nerve. Images were converted using Image J software $(\mathrm{NIH})$ using a set threshold level to allow for the quantification of the immunostained area calculated as percentage area of the width of a normal nerve.

\section{Quantification of nerve regeneration for the long-term experiment}

For the 16-week study, 8- $\mu \mathrm{m}$-thick sections were cut transversely at $3 \mathrm{~mm}$ distally. Immunohistochemistry was carried out to assess myelination using monoclonal mouse antimyelin basic protein (MBP) (Millipore) (dil. 1:500) and polyclonal rabbit anti-PGP9.5 (Dako) (dil. 1:200). Mouse $\mathrm{IgG}, \mathrm{Cy}^{\mathrm{TM}} 3$ linked from goat (GE Healthcare) (dil. 1:500) and fluorescein anti-rabbit $\operatorname{IgG}(\mathrm{H}+\mathrm{L})$ made in goat (Vector Laboratories) (dil. 1:100) were used as secondary antibodies.

For the morphometric analysis, representative fields were selected from the sections ( $n=9$ per nerve). The number of axons, area of axons $\left(\mu \mathrm{m}^{2}\right)$, and area of myelin $\left(\mu \mathrm{m}^{2}\right)$ were assessed using ImageJ software.

\section{Electrophysiology}

Sixteen weeks after nerve transection, the animals were deeply anesthetized and sciatic nerves were fully exposed. Electromyographic assessment was carried out by stimulating proximally to the regenerated nerve using a bipolar stimulatory electrode. A collector needle electrode was placed in the gastrocnemius muscle. The anode electrode was inserted in a tail. The stimulation and recording was made by PowerLab 26T (AD Instruments) and the amplitude of compound muscle action potential (CMAP) (baseline to peak measurements) was assessed and presented as the ratio of operated to control side.

\section{Muscle wet weight}

Medial gastrocnemius muscles of both contralateral and operated sides were weighed and the gastrocnemius muscle weight ratio (operated side/control side) was calculated.

\section{Skin reinnervation analysis}

From each footpad, a biopsy was taken using skin punch biopsy with $5 \mathrm{~mm}$ diameter and embedded in OCT and frozen in liquid nitrogen. Systematic random sections with $15 \mu \mathrm{m}$ thickness were collected onto microscopic slides (SuperFrost ${ }^{\circledR}$ Plus; VWR). Pontamine Sky blue (dil. 1:1000) (Gurr) was used as a nonspecific stain, for $30 \mathrm{~min}$ at room temperature. Then, the sections were incubated with polyclonal rabbit antisera for protein gene product 9.5 (PGP 9.5; dil. 1:500) (Dako) and fluorescein goat anti-rabbit IgG $(\mathrm{H}+\mathrm{L})$ (Vector Laboratories) (dil. 1:100).

Six sections per skin were imaged and analyzed using a fluorescence microscope (BX60; Olympus) and image processing softwares (ImageJ and Image pro Plus) to quantify the nerve fiber area. ${ }^{22}$ The area of regenerated nerve fiber in the operated side was measured and presented as a percentage of the stained area of operated/control side.

\section{Statistical analysis}

GraphPad Prism 5 software (GraphPad Software) was used to run the statistical analysis. T-test and one-way ANOVA followed by Tukey's multiple comparison tests were carried out to analyze the differences across the experimental groups. A value of $p<0.05$ was set as a threshold for significant difference.

\section{Results}

\section{Characterization of the polymer films}

Films had the sufficient mechanical strength and flexibility to be rolled around a mandrel and formed into a tube shape without any cracks or deformation. No significant alteration in groove features was observed in the wet condition. Due to the solvent evaporation method, pits are consistently formed on the surface of the polymer. The mean size of pits on the nongrooved film (pitted film) was $8.42 \pm 3.29 \mu \mathrm{m}^{2}$ with a surface coverage of $6.55 \% \pm 6.41 \%$.

The three-point bending analysis showed that the multilayered conduits (group 3 and 4) displayed a significantly higher flexural rigidity and bending strength in comparison with the thin (group 1) and thick layered conduit (group 2). No significant difference was observed between groups 1 and 2 for flexural rigidity and bending strength (Fig. 2B).

\section{In vitro experiments}

The results of dASC cultures showed that topographical modifications to PCL/PLA surfaces significantly enhanced cell attachment and proliferation. Immunostaining of the cells on the different surface films showed that all PCL/PLA groove substrates provided physical guidance for dASCs, promoting their alignment along the groove surfaces, most notably for SL and V grooves (Fig. 3A). A statistically significant increase in cell attachment was observed in SLgrooved $(p<0.05)$ films when compared with pitted surface films. Interestingly, the cell attachment on the SQ-grooved films did not show any difference from that on pitted surface films (Fig. 3B).

Cell attachment data for the grooved PCL/PLA samples were consistent with the results of the proliferation assay. When dASCs were cultured on either V groove or SL groove substrates, they showed an increased proliferation at both 4 and 8 days compared with cells cultured on SQgrooved and nongrooved films, with a significant difference at both time points $(p<0.001)$ (Fig. 3C). No significant difference was observed for cell proliferation between SL groove and $\mathrm{V}$ groove substrates. Interestingly, on pitted nongrooved films, there was a steady decline of proliferation throughout the time course.

Overall, the results of in vitro tests demonstrated that the surface morphology observed on the grooved PCL/PLA surface affected the cellular response by significantly enhancing cell attachment, alignment, and proliferation. Because of the better results obtained with the SL and V grooves design, these types of films, but not the SQ-grooved films, were used for further in vivo testing. 

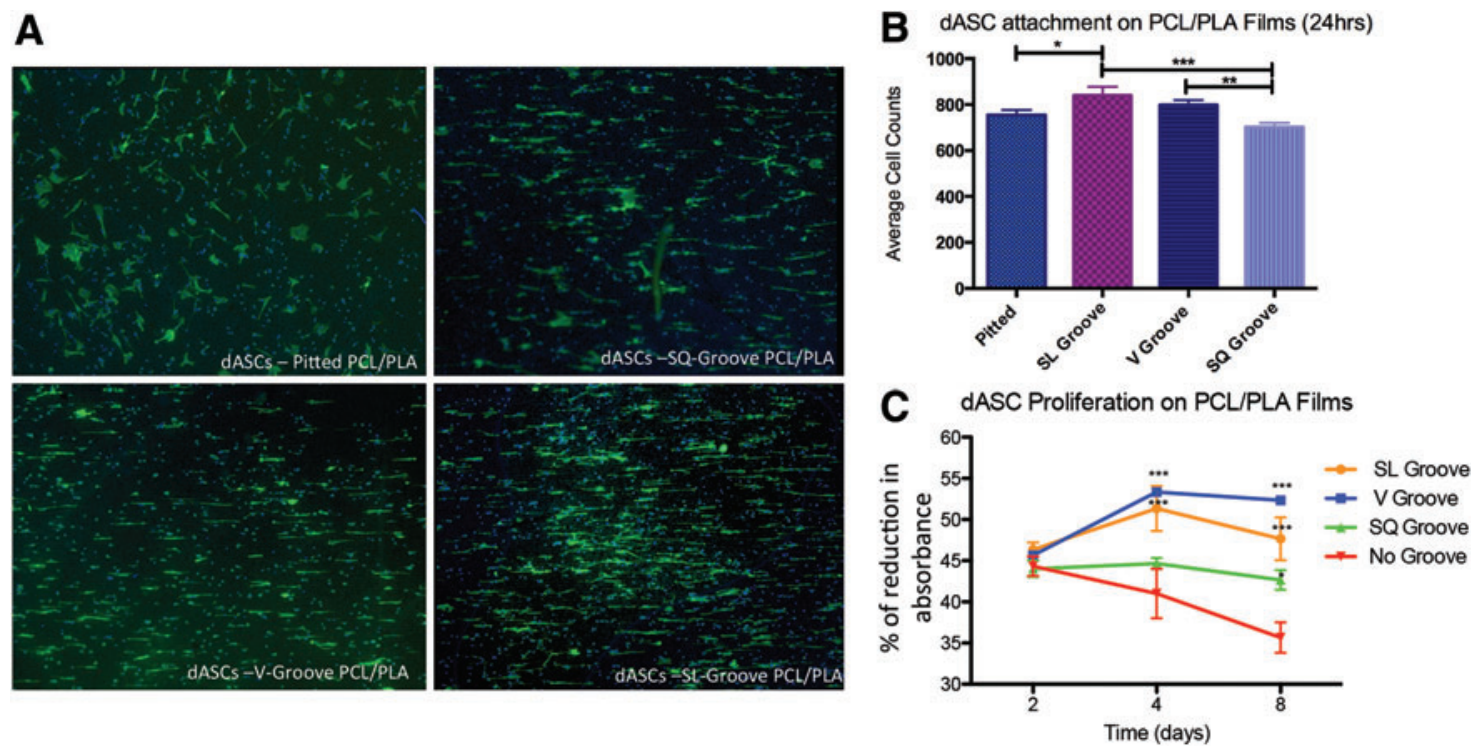

FIG. 3. (A) Cell alignment on PCL/PLA pitted and groove surfaces. Fluorescent images demonstrate that dASCs increase their alignment on V-grooved and SL-grooved surfaces when compared with pitted and SQ-grooved surfaces. Images were taken using a $4 \times$ objective, $* * p<0.01$ and $* * * p<0.001$. (B) Quantitative analysis of dASC attachment to PCL/PLA samples with different surface topography after $24 \mathrm{~h}$ of culture, $n=3,{ }^{*} p<0.05, * * p<0.01$ and $* * * p<0.001$. (C) Results of AlamarBlue $^{\circledR}$ assay, which give an indication of dASC proliferation on PCL/PLA samples over an 8 day culture. $n=3$. ${ }^{*} p<0.05, * * * p<0.001$. dASC, differentiated adipose-derived stem cell; PCL, poly $\varepsilon$-caprolactone; PLA, polylactic acid. Color images available online at www.liebertpub.com/tea

\section{In vivo experiments}

At the time of tissue harvest, the conduits were found to be intact in all groups, and there was no evidence of infection, inflammation, or problematic scar tissue. The structure of the conduit was maintained without deformation or major degradation. The SEM images of the inner surface of the implanted conduits for 3 and 16 weeks showed the intact morphology of the grooved structure (Fig. 4). It was apparent that after 16 weeks of implantation, the conduits started to degrade and lose their integrity by showing a number of small fractures (Fig. 4B).

Short-term (3 weeks) study comparing grooved conduits with nongrooved conduits. Immunostaining of the nerves showed that the regenerating axons had bridged the injury gap in all animals, irrespective of the conduit patterning
(Fig. 5A). There was a statistically significant increase in the amount of regenerating axons, expressed as stained percentage area, in grooved conduits compared with nongrooved controls. This was most noticeable in SL-grooved conduits $(p<0.0001$ vs. nongrooved control) than in $\mathrm{V}$-grooved conduits $(p<0.01$ vs. nongrooved control) (Fig. 5B).

Short-term (3 weeks) study comparing wall thickness of grooved conduits. Given the findings reported above [cf. Short-Term (3 weeks) Study Comparing Grooved Conduits with Nongrooved Conduits section], the SL-grooved conduits were taken forward for a further short-term in vivo experiment to investigate the optimum conduit wall thickness. At harvest, good nerve regeneration was observed in single-layered SL-grooved conduits, and quantification
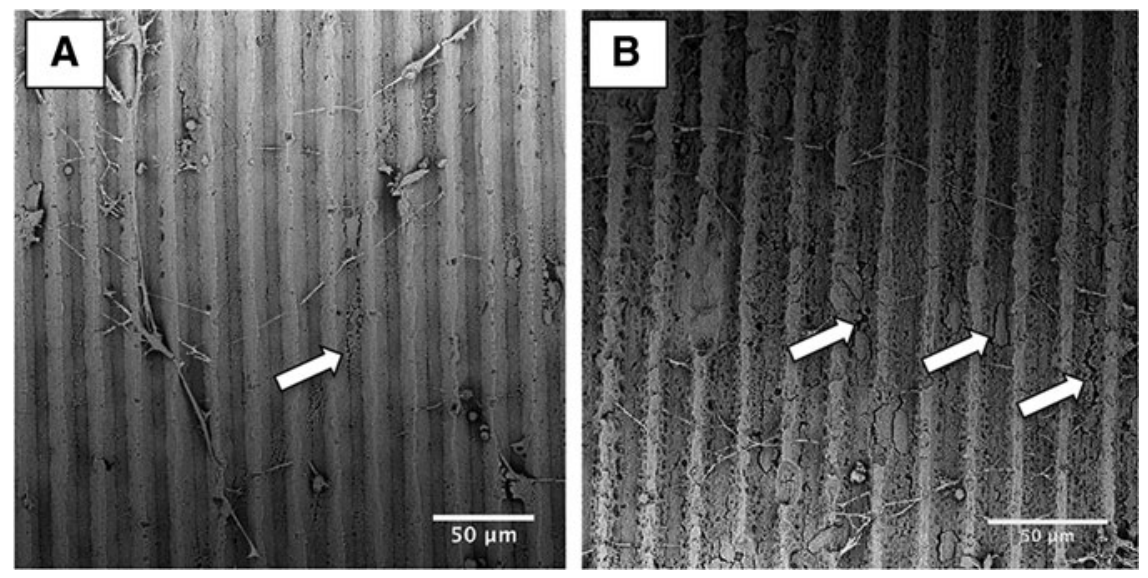

FIG. 4. SEM images of the inner luminal grooved surface. The grooved structure after 3 weeks (A) of implantation was maintained intact; however, the material initiated to degrade after 16 weeks (B) the white arrows show the cracks. 
A
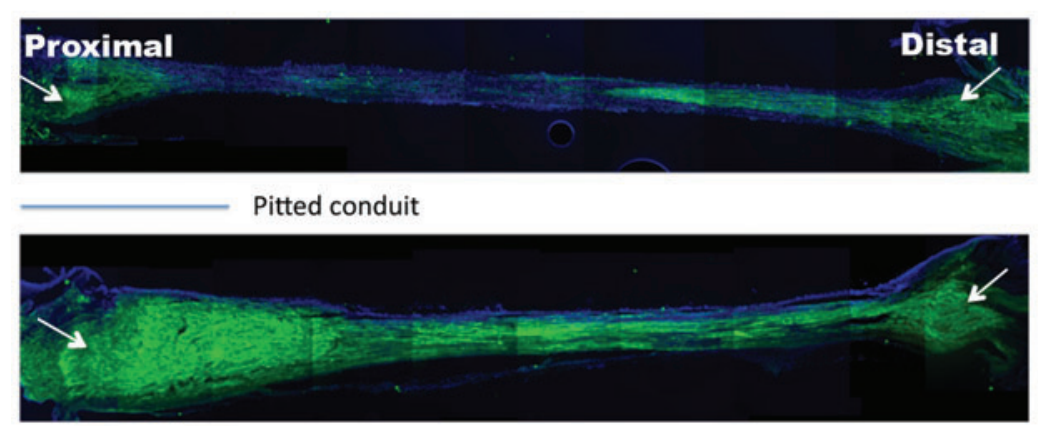
$\square$ SL-grooved conduit

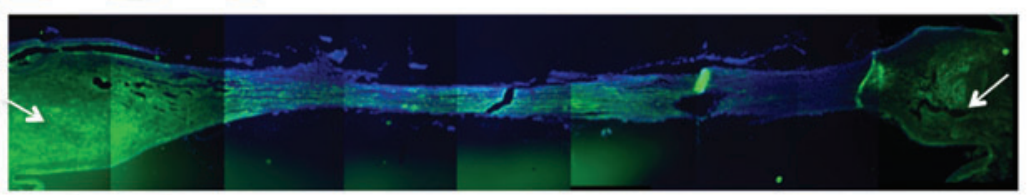
$\bigwedge$ V-grooved conduit

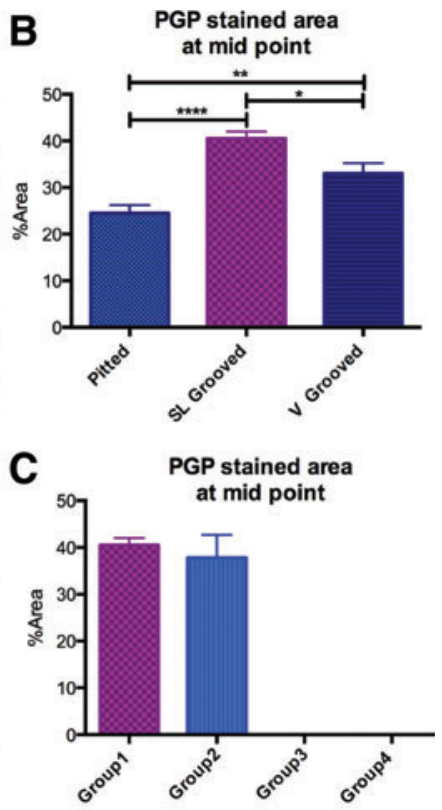

FIG. 5. (A) Overlapping images showing the increased area of PGP9.5 staining of regenerating nerves in grooved conduits compared with nongrooved conduits. (B) The quantification measurement showed an increased area of PGP9.5 staining of regenerating nerve in grooved conduits in thin wall $(70 \mu \mathrm{m})$ conduits compared with nongrooved conduits. $n=4$, $* p<0.05, * * p<0.01$ and $* * * * p<0.0001$. (C) Quantification of nerve regeneration in SL-grooved conduits with different wall thickness (group 1-4) showing the similarity in the area of PGP9.5 staining of regenerating nerves in group 1 and group 2; however, no regeneration was observed in the thick wall conduits in groups 3 and 4. $n=6$. Color images available online at www.liebertpub.com/tea

showed that there was no statistical difference between the thin and thick single-layered conduits (group 1 and 2; Fig. 5C). On macroscopical analysis, regeneration was absent in 3 animals grafted with the rolled spiral layered conduits (group 3), and was minimal in the remaining 3 animals. Similarly, in 5 animals grafted with the multilayered conduits (group 4), there was no regeneration with very small amount of regeneration in the remaining animal (Fig. 5C). No further analysis was undertaken for these two groups.

Short-term (3 weeks) and long-term (16 weeks) studies comparing the SL-grooved conduit with autograft. Nerve regeneration analysis: In all experimental groups, the axons regenerated across the 10-mm gap after 3 weeks. The axonal density, which was evaluated by measuring the PGP-positivestained area at the distal stump, showed no significant dif- ference between the autograft $(\mathrm{Ag})(48.56 \% \pm 16.33 \%)$ and conduit (C) $(36.32 \% \pm 12.13 \%)$ group (Fig. 6).

The analysis of regenerated nerves following 16 weeks of nerve transection assessed the features of axonal regeneration and maturation of myelin sheath (Fig. 7A). Quantitative analysis evaluated number of axons, area of axons, and area of myelin (Fig. 7B). The $\mathrm{C}$ group showed comparable values with the Ag group in area of axons $\left(137.9 \pm 43.9 \mu \mathrm{m}^{2}\right.$ vs. $\left.184.9 \pm 47.79 \mu \mathrm{m}^{2}\right)$, number of axons $(96.2 \pm 14.5$ vs. $107 \pm$ $15.72)$, and area of myelin $\left(215.5 \pm 95.08 \mu \mathrm{m}^{2}\right.$ vs. $349.2 \pm$ $161.9 \mu \mathrm{m}^{2}$ ) (Fig. 7B).

Electrophysiology: The CMAP was measured on lateral and medial gastrocnemius muscles 16 weeks after nerve transection (Fig. 8A, B). The recovery of CMAP amplitude (operated: control side ratio) in lateral and medial gastrocnemius muscles showed no significant difference between
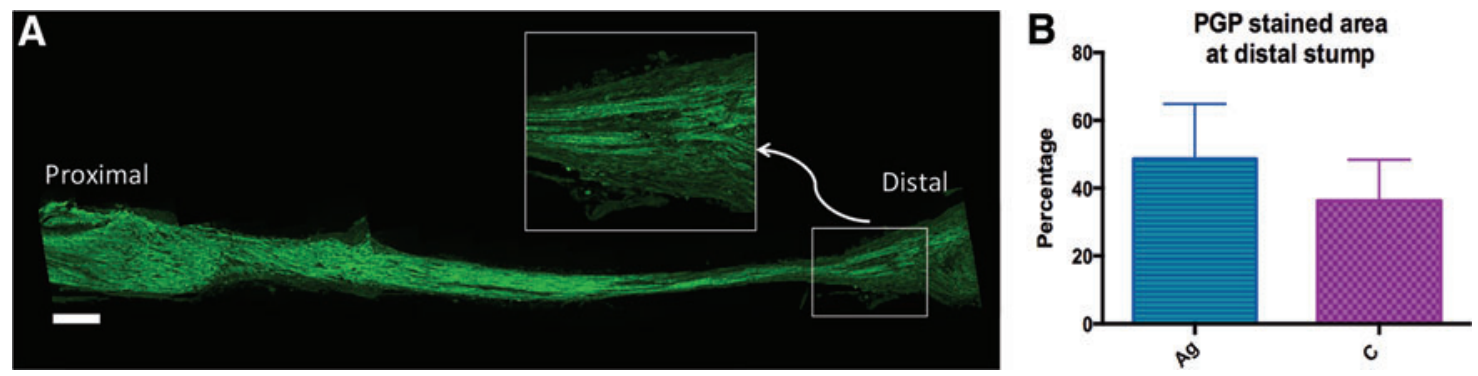

FIG. 6. (A) Regenerated nerves were stained with PGP9.5. The inset images show the distal stump in higher magnification. The scale bar shows $500 \mu \mathrm{m}$. (B) Quantitative analysis of nerve regeneration following 3 weeks of nerve transection at the distal stump. No significant difference was observed comparing the Ag group and C group. Ag, autograft; C, conduit. Color images available online at www.liebertpub.com/tea 
A

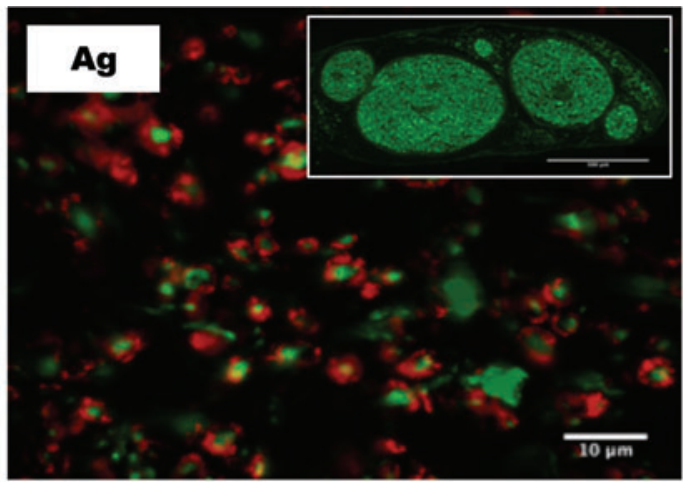

B

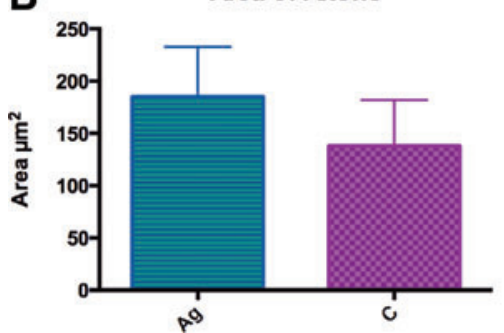

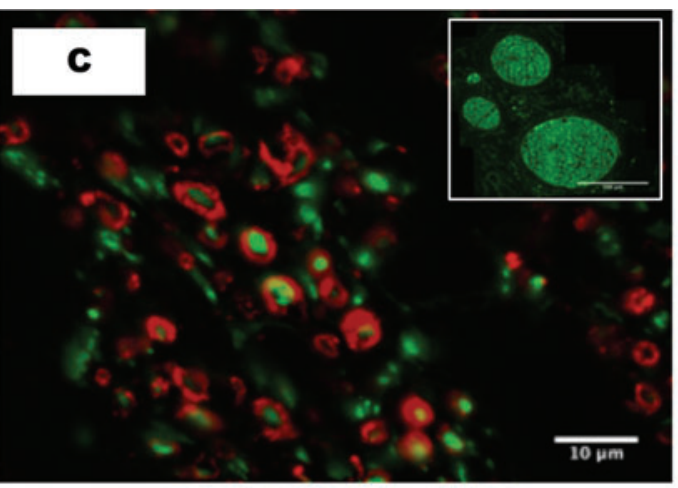

Area of myelin
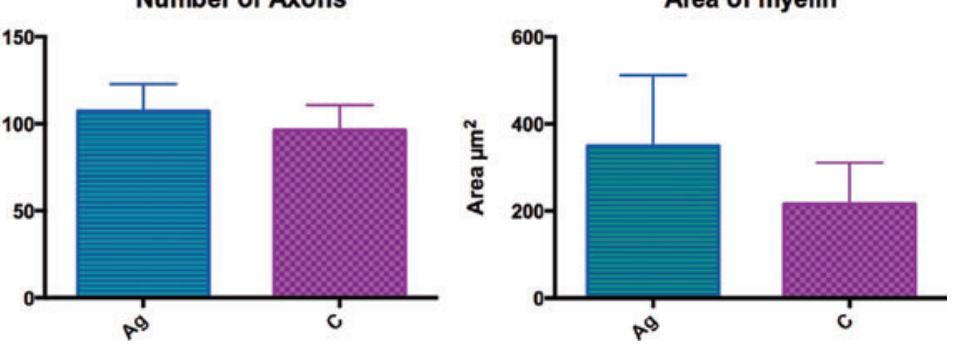

FIG. 7. Immunostaining images and measurement of regenerated nerves following 16 weeks of nerve transection at $3 \mathrm{~mm}$ distally. (A) Myelin was stained by MBP and axon was stained by PGP. The inset images show the cross section of the nerve for $\mathrm{C}$ and $\mathrm{Ag}$ groups. (B) The number of axons, area of axons, and area of myelin were calculated at $3 \mathrm{~mm}$ distally. The nerve regeneration features in the $\mathrm{C}$ group were comparable with the $\mathrm{Ag}$ group. $\mathrm{MBP}$, myelin basic protein. Color images available online at www.liebertpub.com/tea

the $\mathrm{C}$ group and $\mathrm{Ag}$ group $(0.243 \pm 0.054$ vs. $0.257 \pm 0.051$ and $0.412 \pm 0.274$ vs. $0.233 \pm 0.114$, respectively).

Muscle wet weight: A decrease in muscle mass is usually observed following the removal of neural innervation before recovering mass with reinnervation. This effect was measured with the medial gastrocnemius muscle wet weight harvested after 16 weeks and was expressed as a ratio of the control side (Fig. 8C). No significant difference was detected between the $\mathrm{C}$ group $(63 \% \pm 7.13 \%)$ and $\mathrm{Ag}$ group $(58 \% \pm 7.01 \%)$.

Skin reinnervation: The reinnervation of the footpads of both operated and control sides was measured. The PGPpositive-stained area was measured and the ratio of the operated to control side was calculated. The percentage of
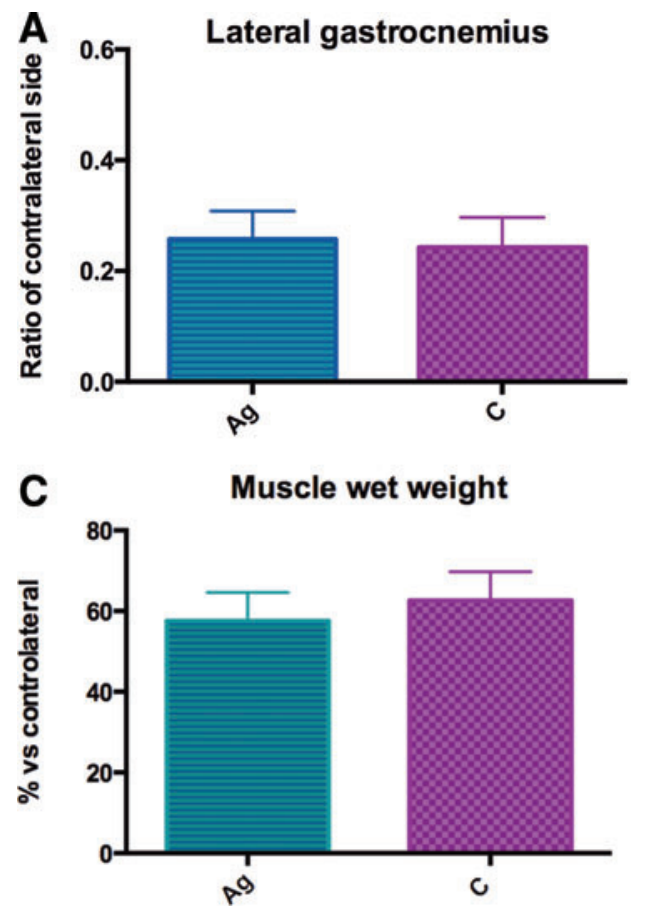
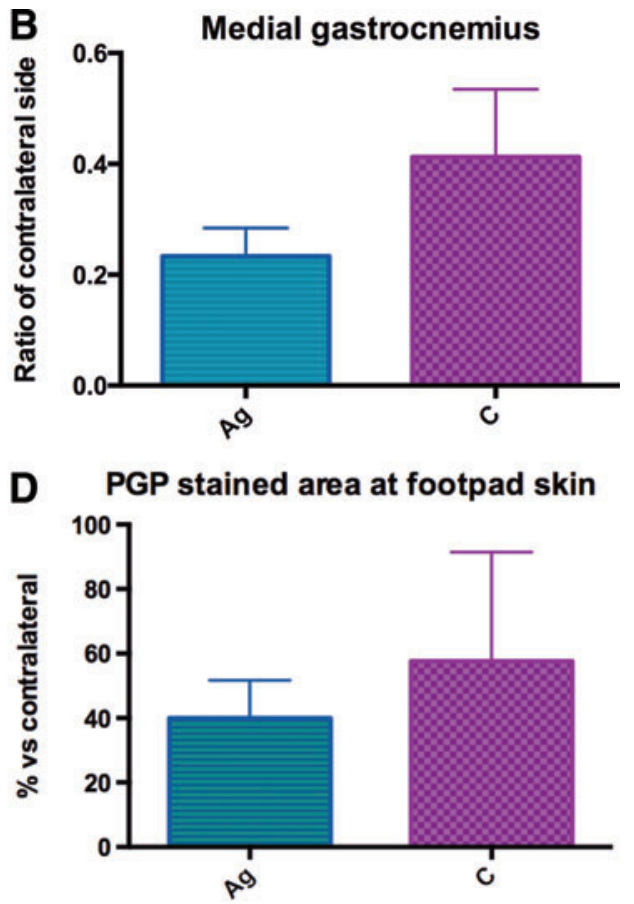

FIG. 8. CMAP recovery of (A) lateral and (B) medial gastrocnemius muscles was measured following 16 weeks of nerve transection. No significant difference was indicated in CMAP recovery of lateral and medial muscles between $\mathrm{C}$ and $\mathrm{Ag}$ groups; (C) Wet muscle weight was measured after 16 weeks of nerve transection. No significant difference was indicated between $\mathrm{C}$ and $\mathrm{Ag}$ groups. (D) Footpad skin reinnervation was measured following 16 weeks of nerve transection. The percentage of reinnervation for $\mathrm{Ag}$ and $\mathrm{C}$ groups indicated no significant difference. CMAP, compound muscle action potential. Color images available online at www .liebertpub.com/tea 
nerve fiber reinnervation indicated no significant difference between the $\mathrm{C}$ group $(57.62 \% \pm 33.37 \%)$ and $\mathrm{Ag}$ group $(39.97 \% \pm 11.75 \%)$ (Fig. 8D).

\section{Discussion}

The biodegradable nerve guide scaffold proved to be a potential treatment to promote peripheral nerve injury recovery. ${ }^{23}$ An effective bioengineered nerve conduit should provide a supportive environment to both the regenerating axons and the proliferating Schwann cells. In addition, the biomechanical and physical properties of the polymeric conduit should complement the mechanical properties of the surrounding tissues to facilitate the regeneration process. Following the implantation, neural cells use a scaffold as a platform for adhesion, migration, and proliferation. ${ }^{24}$ The biocompatibility of polyesters (e.g., PCL, PLA, or PGA) used in medical devices are well studied. ${ }^{25}$ The materialhost surrounding tissue and cells interaction involves complex responses from both sides. At the macroscopic level, cellular attachment on the surface of biomaterial is controlled by the surface charge, hydrophilicity, and physiochemical properties of the surface. ${ }^{26}$ The concept that cells respond to the topographical features of their environment has been observed in many different studies, ${ }^{7,27,28}$ and various responses have been observed in terms of cellular adhesion, proliferation, and morphology. ${ }^{29}$ In previous studies, we have demonstrated an improved cellular response to the modification of the biomaterial surface with microscale pits, ${ }^{2}$ most likely due to the increased surface roughness, which improves the cell attachment, and which may mimic the surface topography formed by the extracellular matrix (ECM) components of the basal lamina. This could potentially affect the endogenous Schwann cells in producing neurotrophic factors, such as nerve growth factor and brained-derived neurotrophic factor, and ECM molecules, such as laminin, which promote and support nerve regeneration. $^{30}$

Studies on microgroove topography have indicated that in neuronal cell cultures neurite alignment increases with increasing groove depth from 1 to $4 \mu \mathrm{m},{ }^{31}$ while little effect is observed on feature sizes less than $300 \mathrm{~nm}^{32}$ Schwann cells can also be guided by topography and like neurites appear to be sensitive to the depth of groove topography, ${ }^{33}$ indicating that there is a synergy between the cues presented by cells and the cues presented by the substrate topography. Our previous study showed that neural cells attached and oriented along the PCL/PLA-grooved surfaces, becoming elongated and highly polarized during cell spreading and showing an ordered alignment. ${ }^{21}$ Consistently, the in vitro results of this study showed that dASCs assumed a spindleshaped Schwann cell-like morphology within the PCL/PLA grooves, and they aligned longitudinally to resemble the bands of Büngner. These cellular structures are found in damaged nerves following Wallerian degeneration and provide an essential pathway to guide the axonal growth. ${ }^{34}$

We were also able to demonstrate an improved cell proliferation on films with SL grooves; consequently, an increased number of cells might result in increased secretion of neurotrophic factors and ECM molecules. The proliferation on SL grooves was significantly better than on SQ-grooved films, indicating that the surface topology is important for cell attachment and proliferation. Although in vitro cell proliferation experiments indicated that there was little difference between SL and V-grooved films, these results were not reflected during the in vivo testing of the groove conduits, as SL-grooved conduits stimulated improved nerve regeneration. It can be hypothesized that the presence of ridges on the SL-grooved films, compared with their absence in V-grooved films, has a significant influence on the behavior of glial cells. During in vivo experiments, this would result in increased migration of Schwann cells from the nerve stump, resulting in the improved regeneration seen in this study, consistent with previous findings. ${ }^{35}$

The topographical modifications of PCL/PLA conduits, mimicking the architectural surface topography of the natural tissue, may act to induce cellular responses from both the endogenous Schwann cells and regenerating neurons, resulting in the significant improvement of nerve regrowth observed for the microgrooved PCL/PLA conduits when compared with the nongrooved PCL/PLA conduit controls. These observations would be explained by the fact that in vitro dASCs have also been observed to align in a highly polarized manner along the grooves, which may also happen for Schwann cells in the inner lumen of the conduits, possibly due to the topographical guidance cues in the material surfaces. Nerve regeneration through micropitted conduits was seen in previous studies, ${ }^{2}$ and the present results show an improvement of the regeneration obtained using grooved PCL/PLA conduits, pointing to the effects of surface morphology on the regenerating cells. Our results are also consistent with those of other groups, who showed that PLA conduits with microgrooves on the inner surface improved peripheral nerve regeneration when compared with smooth conduit controls. $^{13}$

Experiments with thicker wall conduits were carried out and the results showed that a limited increase of wall thickness did not affect the regeneration results. However, the increased thickness was effective only for single-layered conduits as the use of multilayered or spiral conduits resulted in reduced or absent regeneration. This result was unexpected, and it can be speculated that is may be due to the fact that the increased wall thickness could limit the permeability of the conduit, which is a substantial factor for exchange of metabolic elements, diffusion of nutrients, and oxygen. $^{23}$ The increased rigidity could be the other factor for the failure of the conduit with increased wall thickness. Balanced strength and flexibility are required for the neural scaffold to resist pressure from handling, suturing, and surrounding tissue, while too rigid material could damage nerve stumps or surrounding tissues. ${ }^{23}$

The results of the current study indicated that the grooved PCL/PLA conduits showed a comparable experimental outcome to autografts. The grooved PCL/PLA conduits demonstrated improved nerve regeneration compared with previously published data on alternative conduits in similar experimental models. ${ }^{36-38}$ Furthermore, similar end organ reinnervation, wet muscle weight, and CMAP amplitude were observed in the long-term experiment.

This investigation addresses the application of biodegradable polymer conduits with an intraluminal structure to be used in peripheral nerve repair. The promising outcomes reported herein suggest that the grooved PCL/PLA conduits could be a potential alternative to nerve grafting. 


\section{Acknowledgments}

The authors would like to thank the National Institute for Health Research (NIHR), Invention for Innovation (i4i) Programme for financial support. This article presents independent research commissioned by the NIHR under the i4iProgramme. The views expressed in this publication are those of the authors and not necessarily those of the NHS, the NIHR, or the Department of Health. The authors would like to thank Acorda Therapeutics, for supplying GGF-2 for the culture of differentiated stem cells. A.R. is supported by the National Institute for Health Research, Academy of Medical Sciences, and the British Society for Surgery of the Hand.

\section{Disclosure Statement}

No competing financial interests exist.

\section{References}

1. Li, J.M., and Shi, R.Y. Fabrication of patterned multiwalled poly-L-lactic acid conduits for nerve regeneration. J Neurosci Methods 165, 257, 2007.

2. Sun, M., Kingham, P.J., Reid, A.J., Armstrong, S.J., Terenghi, G., and Downes, S. In vitro and in vivo testing of novel ultrathin PCL and PCL/PLA blend films as peripheral nerve conduit. J Biomed Mater Res A 93, 1470, 2010.

3. Evans, G.R.D., Brandt, K., Widmer, M., Lu, L., Meszlenyi, R.K., Gupta, P.K., et al. In vivo evaluation of poly(l-lactic acid) porous conduits for peripheral nerve regeneration. Biomaterials 20, 1109, 1999.

4. Bastioli, C., ed. Handbook of biodegradable polymer. Shawbury, UK: Smithers Rapra Technology 2005, p. 549.

5. Sun, M., McGowan, M., Kingham, P.J., Terenghi, G., and Downes, S. Novel thin-walled nerve conduit with microgrooved surface patterns for enhanced peripheral nerve repair. J Mater Sci Mater Med 21, 2765, 2010.

6. Tse, K.H., Sun, M., Mantovani, C., Terenghi, G., Downes, S., and Kingham, P.J. In vitro evaluation of polyester-based scaffolds seeded with adipose derived stem cells for peripheral nerve regeneration. J Biomed Mater Res Part A 95A, 701, 2010.

7. Curtis, A., and Wilkinson, C. Topographical control of cells. Biomaterials 18, 1573, 1997.

8. Chen, C.S., Mrksich, M., Huang, S., Whitesides, G.M., and Ingber, D.E. Geometric control of cell life and death. Science 276, 1425, 1997.

9. Dalby, M.J., Riehle, M.O., Yarwood, S.J., Wilkinson, C.D.W., and Curtis, A.S.G. Nucleus alignment and cell signaling in fibroblasts: response to a micro-grooved topography. Exp Cell Res 284, 274, 2003.

10. Biggs, M.J.P., Richards, R.G., and Dalby, M.J. Nanotopographical modification: a regulator of cellular function through focal adhesions. Nanomed Nanotechnol Biol Med 6, 619, 2010.

11. Miller, C., Jeftinija, S., and Mallapragada, S. Micropatterned Schwann cell-seeded biodegradable polymer substrates significantly enhance neurite alignment and outgrowth. Tissue Eng 7, 705, 2001.

12. Miller, C., Shanks, H., Witt, A., Rutkowski, G., and Mallapragada, S. Oriented Schwann cell growth on micropatterned biodegradable polymer substrates. Biomaterial 22, 1263, 2001.
13. Hsu, S.H., and Ni, H.C. Fabrication of the microgrooved/ microporous polylactide substrates as peripheral nerve conduits and in vivo evaluation. Tissue Eng Part A 15, 1381, 2009.

14. Mosahebi, A., Woodward, B., Wiberg, M., Martin, R., and Terenghi, G. Retroviral labeling of Schwann cells: in vitro characterization and in vivo transplantation to improve peripheral nerve regeneration. Glia 34, 8, 2001.

15. Madduri, S., and Gander, B. Schwann cell delivery of neurotrophic factors for peripheral nerve regeneration. J Peripher Nerv Syst 15, 93, 2010.

16. Mosahebi, A., Fuller, P., Wiberg, M., and Terenghi, G. Effect of allogeneic Schwann cell transplantation on peripheral nerve regeneration. Exp Neurol 173, 213, 2002.

17. Hadlock, T.E.J. A tissue-engineered conduit for peripheral nerve repair. Arch Otolaryngol Neck Surg 124, 1081, 1998.

18. Tohill, M., and Terenghi, G. Stem-cell plasticity and therapy for injuries of the peripheral nervous system. Biotechnol Appl Biochem 40, 17, 2004.

19. Kingham, P.J., Kalbermatten, D.F., Mahay, D., Armstrong, S.J., Wiberg, M., and Terenghi, G. Adipose-derived stem cells differentiate into a Schwann cell phenotype and promote neurite outgrowth in vitro. Exp Neurol 207, 267, 2007.

20. Di Summa, P.G., Kingham, P.J., Raffoul, W., Wiberg, M., Terenghi, G., and Kalbermatten, D.F. Adipose-derived stem cells enhance peripheral nerve regeneration. J Plast Reconstr Aesthet Surg 63, 1544, 2010.

21. Mobasseri, S.A., Terenghi, G., and Downes, S. Microstructural geometry of thin films intended for the inner lumen of nerve conduits affects nerve repair. J Mater Sci Mater Med 24, 1639, 2013.

22. Hart, A.M., Wilson, A.D.H., Montovani, C., Smith, C., Johnson, M., Terenghi, G., et al. Acetyl-l-carnitine: a pathogenesis based treatment for HIV-associated antiretroviral toxic neuropathy. AIDS Lond Engl 18, 1549, 2004.

23. Ichihara, S., Inada, Y., and Nakamura, T. Artificial nerve tubes and their application for repair of peripheral nerve injury: an update of current concepts. injury 39, S29, 2008.

24. Gu, X., Ding, F., and Williams, D.F. Neural tissue engineering options for peripheral nerve regeneration. Biomaterials 35, 6143, 2014.

25. Jiang, X., Lim, S.H., Mao, H.-Q., and Chew, S.Y. Current applications and future perspectives of artificial nerve conduits. Exp Neurol 223, 86, 2010.

26. Shi, D. Biomaterials and Tissue Engineering. Beijing, China: Springer Science \& Business Media, 2004, p. 268.

27. Mitchel, J.A., and Hoffman-Kim, D. Cellular scale anisotropic topography guides Schwann cell motility. PLoS One 6, e24316, 2011.

28. Zhou, F., Yuan, L., Huang, H., and Chen, H. Phenomenon of "contact guidance" on the surface with nano-microgroove-like pattern and cell physiological effects. Chin Sci Bull 54, 3200, 2009.

29. Martinez, E.C., Ivirico, J.L.E., Criado, I.M., Ribelles, J.L.G., Pradas, M.M., and Sanchez, M.S. Effect of poly(Llactide) surface topography on the morphology of in vitro cultured human articular chondrocytes. J Mater Sci Mater Med 18, 1627, 2007.

30. Terenghi, G. Peripheral nerve regeneration and neurotrophic factors. J Anat 194, 1, 1999.

31. Miller, C., Jeftinija, S., and Mallapragada, S. Synergistic effects of physical and chemical guidance cues on neurite 
alignment and outgrowth on biodegradable polymer substrates. Tissue Eng 8, 367, 2002.

32. Clark, P., Connolly, P., Curtis, A.S., Dow, J.A., and Wilkinson, C.D. Topographical control of cell behaviour: II. Multiple grooved substrata. Development 108, 635, 1990.

33. Hsu, S., Chen, C., Lu, P.S., Lai, C., and Chen, C. Oriented Schwann cell growth on microgrooved surfaces. Biotechnol Bioeng 92, 579, 2005.

34. Hall, S. Axonal regeneration through acellular muscle grafts. J Anat 190, 57, 1997.

35. Hsu, S., Lu, P.S., Ni, H.-C., and Su, C.-H. Fabrication and evaluation of microgrooved polymers as peripheral nerve conduits. Biomed Microdevices 9, 665, 2007.

36. Erba, P., Mantovani, C., Kalbermatten, D.F., Pierer, G., Terenghi, G., and Kingham, P.J. Regeneration potential and survival of transplanted undifferentiated adipose tissuederived stem cells in peripheral nerve conduits. J Plast Reconstr Aesthet Surg JPRAS 63, e811, 2010.

37. Di Summa, P.G., Kalbermatten, D.F., Pralong, E., Raffoul, W., Kingham, P.J., and Terenghi, G. Long-term in vivo regeneration of peripheral nerves through bioengineered nerve grafts. Neuroscience 181, 278, 2011.

38. Di Summa, P.G., Kingham, P.J., Campisi, C.C., Raffoul, W., and Kalbermatten, D.F. Collagen (NeuraGen $\left.{ }^{\circledR}\right)$ nerve conduits and stem cells for peripheral nerve gap repair. Neurosci Lett 572, 26, 2014.

Address correspondence to:

Giorgio Terenghi, PhD

Blond McIndoe Laboratories

Centre for Tissue Injury and Repair Institute of Inflammation \& Repair

University of Manchester Manchester M13 9PT United Kingdom

E-mail: giorgio.terenghi@manchester.ac.uk

Received: May 8, 2014

Accepted: November 5, 2014

Online Publication Date: January 16, 2015 